\title{
On the Coronal Current-Free Global Field Configuration
}

\author{
Yihua Yan \\ National Astronomical Observatories, Chinese Academy of Sciences, A20 Datun Road, \\ Chaoyang District, Beijing 100012, China \\ email:yyh@bao.ac.cn
}

\begin{abstract}
A coronal current-free field model, which applies the asymptotic condition of no field at infinity and the boundary condition on the solar surface specified, is presented. Some Applications of the method to practical solar events indicate that the extrapolated global magnetic field structures effectively demonstrate the case for the disk signature of the radio CME and the evolution of the radio sources during the CME/flare processes.
\end{abstract}

Keywords. Sun: corona, Sun: coronal mass ejections (CMEs), Sun: magnetic fields

\section{Introduction}

The coronal magnetic field configuration is important for understanding the energy storage and release processes that account for CMEs. The source-surface is generally employed to reconstruct the coronal field configuration by fitting the observed coronal structures and a parameter is needed in order to obtain closed field region versus open field lines for the source-surface method or its variants (Hoeksema \& Scherrer (1986), Zhao \& Hoeksema (1994)). Here we present a model which is based on the work for potential magnetic field problems that only applies the condition at infinity with the boundary condition on the solar surface specified (Yan, Yu \& Shi (1993), or recently in Wang, Yan, Wang, et al. (2002)). For some event analyses, we have employed MDI/SOHO longitudinal magnetogram inserted into the synoptic magnetogram to obtain whole boundary condition over the solar surface. Due to the projection effect, we employed MDI magnetogram data in the central region of $\pm 50^{\circ}$ longitude and $\pm 50^{\circ}$ latitude and the MDI synoptic magnetogram data in the regions of $-180^{\circ}$ to $-50^{\circ}$ and $50^{\circ}$ to $180^{\circ}$ longitude, and $\pm 60^{\circ}$ latitude. The extrapolated global magnetic field structures effectively demonstrate the case for the disk signature of the radio $\mathrm{CME}$ and the evolution of the radio sources during the CME/flare processes.

\section{Method}

The extrapolation code is based on the work for potential magnetic field problems (Yan, Yu \& Shi (1993); e.g., recently in Wang, Yan, Wang, et al. (2002)). For the potential condition, or current-free field, which is governed by $\nabla \times \mathbf{B}=0$ and $\nabla \cdot \mathbf{B}=0$, the magnetic field can be represented by a scalar potential $\Psi$ with $\mathbf{B}=-\nabla \Psi$ Then we have the Laplacian equation.

$$
\nabla^{2} \Psi=0 .
$$

On the solar surface $S$ we have line-of-sight field component or its normal component $B_{n}$ specified, i.e.,

$$
B_{n}=-\frac{\partial \Psi}{\partial n} .
$$


Therefor we can obtain a boundary value problem of (2.1-2.2), e.g., as described in Sakurai (1989). In general, the potential $\Psi$ at any position $\mathbf{r}_{i}$, in space $V$ can be determined from (Courant \& Hilbert 1962):

$$
\Psi\left(\mathbf{r}_{i}\right)=\oint_{S}\left[G\left(\mathbf{r}_{i} ; \mathbf{r}\right) \frac{\partial \Psi(\mathbf{r})}{\partial n}-\frac{\partial G\left(\mathbf{r}_{i} ; \mathbf{r}\right)}{\partial n} \Psi(\mathbf{r})\right] d S
$$

where $G=1 / 4 \pi\left|\mathbf{r}_{i}-\mathbf{r}\right|$ is Green's function of Laplacian equation in free space. The Green's function solution of the above boundary value problem was applied to practical solar magnetic field observations in early 1960s (Sakurai (1989)). Here, the magnetic field is, however, obtained from:

$$
\mathbf{B}=\oint_{S}\left[\Psi \frac{\partial}{\partial r}\left(\frac{\partial G}{\partial n}\right)-\frac{\partial \Psi}{\partial n} \frac{\partial G}{\partial r}\right] d s
$$

with $\partial \Psi / \partial n$ known over the boundary and $\Psi$ solved numerically by the boundary element method (Yan, Yu \& Shi (1993)), which is a well-established method for science and technology applications (Brebbia, Telles \& Wrobel (1984)).

\section{Results}

For the event analysis, we have employed MDI/SOHO longitudinal magnetogram inserted into the synoptic magnetogram to obtain whole boundary condition over the solar surface. Due to the projection effect, we employed MDI magnetogram data in the central region of $\pm 50^{\circ}$ longitude and $\pm 50^{\circ}$ latitude and the MDI synoptic magnetogram data in the regions of $-180^{\circ}$ to $-50^{\circ}$ and $50^{\circ}$ to $180^{\circ}$ longitude, and $\pm 60^{\circ}$ latitude. The method has been applied in Wang, Yan, Wang, et al. (2002) for a trans-equatorial CME event in May 1998.

For the 17-Mar-2002 event, the extrapolated global magnetic field structures effectively demonstrate the case for the disk signature of the radio CME and the evolution of the radio sources during the $\mathrm{CME} /$ flare processes. It is obtained that the radio counterpart of the CME as well as source of type III burst was propagating along the open field lines. The result shows that the extrapolated field structures are very helpful to understand the flare/CME process. The detailed analysis is to be presented elsewhere separately.

\section{Acknowledgements}

The work is supported by CAS, NSFC grants 10225313, 10333030, and MOST grant G2000078403.

\section{References}

Brebbia, C. A., Telles, J. C. F., \& Wrobel, L. C. 1984, Boundary Element Techniques (Berlin: Springer)

Courant, R. \& Hilbert, D. 1962, Methods of Mathematical Physics (New York: Wiley), Vol. 2

Hoeksema, J. T. \& Scherrer, P. H. 1986, Sol. Phys. 105, 205

Sakurai, T. 1989, Space Sci. Rev. 51, 11

Wang, T. J, Yan, Y. H., Wang, J. L., Kurokawa, H., \& Shibata, K. 2002, ApJ 572, 280

Yan, Y. H. \& Sakurai, T. 2000, Sol. Phys. 195, 89

Yan, Y. H., Yu, Q., \& Shi, H. L. 1993, in: J.H. Kane, G. Maier, N. Tosaka \& S. N. Atluri (eds.), Advance in Boundary Element Techniques (New York: Springer), p. 447

Zhao, X. P. \& Hoeksema, J. T. 1994, Sol. Phys. 151, 91 